ULB-TH-01/24

UNIL-IPT-01-14

\title{
Neutrino masses with a single generation in the bulk.
}

\author{
J.-M. Frère ${ }^{1}$, M.V. Libanov ${ }^{2}$ and S.V. Troitsky ${ }^{2,3}$ \\ 1 Service de Physique Théorique, CP 225, \\ Université Libre de Bruxelles, B-1050, Brussels, Belgium; \\ 2 Institute for Nuclear Research of the Russian Academy of Sciences, \\ 60th October Anniversary Prospect 7a, 117312, Moscow, Russia; \\ 3 Institute of Theoretical Physics, University of Lausanne, \\ CH-1015, Lausanne, Switzerland
}

\begin{abstract}
In a class of multidimensional models, topology of the thick brane provides three chiral fermionic families with hierarchical masses and mixings in the effective four-dimensional theory, while the full model contains a single vectorlike generation. We discuss how to incorporate three non-degenerate neutrino masses in these models with the help of only one singlet bulk fermion.
\end{abstract}




\section{Introduction}

One of the interesting possibilities which open up in theories with more than four spacetime dimensions is to explain the misterious pattern of fermion mass hierarchies [1, 2, 3]. In previous works [2, 3], we have shown how a single family of fermions, with vectorlike couplings to the Standard Model group $S U(3) \times S U(2) \times U(1)$ in 6 dimensions was reduced to $n$ chiral families in 4 dimensions. This mechanism hinged on a localisation on a vortex in the "transverse" dimensions, characterized by a winding number $n$. As a variant, an effective vortex achieving the same result could be simulated by coupling the fermions to a winding-number 1 scalar elevated to the $n$th power.

The value $n=3$ is not favoured in such schemes, and was only invoked for its phenomenological interest. We also showed how a relatively simple scalar structure (the usual Higgs doublet $H$, supplemented by the vortex-defining field $\Phi$ ) suffices to generate the mass hierarchy of the $n$ families, while an auxiliary field $X$ could be used to generate a mixing taking place mostly between adjacent generations.

The key to the construction is that interaction with a vortex with winding number $n$ leads, by the index theorem [四], to $n$ chiral zero modes in four dimensions, and this for each fermion species coupled to the structure.

We now return to this construction and pay special attention to the case of neutrinos. The simplest attitude (which was in a way implicit in the previous papers) would be to treat neutrinos just like the other fermions, that is to include both leftand right-handed fields, say $\nu$ and $N$ in four dimensions, and to generalize them to six dimensions. Dirac masses and mixings are then obtained just like those of the charged fermions. There is nothing fundamental against this approach, except the very small value of the ratio between neutrino and charged fermion masses (typically less than $10^{-6}$ ). This would require severe fine tuning, either directly at the level of the Yukawa couplings themselves, or in a more covert fashion through a more complicated scalar structure (see footnote below).

We therefore try to explore other ways of getting small neutrino masses.

It has become standard practice [5] in the context of extra (flat and compactified) dimensions, to assume that right-handed neutrions, being gauge singlets, could 
propagate in the "bulk" of space, escaping confinement?. This is of course possible since such particles do not participate directly in the gauge interactions, and therefore do not affect (at least at tree level, see for instance Ref. [7]) the behaviour of electroweak exchanges. In such a context, the smallness of the neutrino mass stems not from artficially tuned Yukawa couplings, but from the severely reduced overlap between the confined and "bulk" wave functions. Could such a strategy work here? The transcription of such a mechanism in our context involves the introduction of a six-dimensional fermion field, $N$, uncoupled to the vortex field $\Phi$. This however is in general not sufficient, if the two-dimensional transverse space stays unbounded (a point which we did not really need to specify this far, since the vortex achieved in any case the localisation of physical fields in a four-dimensional tube). Using $\theta$ and $r$ as variables to describe the two extra dimensions, we should now require $r<R$, while keeping $R \gg r_{\text {vortex }}$, to avoid perturbing the previous construction. Is this step sufficient? In principle, if $1 / R$ were close to the expected light neutrino mass scale (say a fraction of an eV), we would have a whole set of Kaluza-Klein towers at hand, each with relatively light states to couple to the $n$ left-handed neutrinos. This line is however somewhat dangerous, since it may lead to a conflict with data on the evolution of supernovae [8]. We will avoid this situation by requesting $1 / R \gg m_{\nu}$. For simplicity we may even takel $1 / R \gg 100 \mathrm{GeV}$.

This further requirement however leads us to a pecular situation: we may not have enough light "right-handed" partners to provide masses to the $n$ neutrinos! Indeed, we typically expect only one fundamental mode of zero mass. Introducing $n$ such fermions would of course solve the problem, but would be in opposition to the approach, where at least the charged family multiplication simply results from the topological structure. Fortunately, even with the simplest use of one $N$ field, we get easily two light states (this is a funciton of the chosen charge assignements, see

${ }^{*}$ Other possibilities include putting left- and right-handed neutrinos to very different places inside the thick brane [6] or putting the scalar (not the fermion) in the bulk. The latter approach can be easily implemented in the model of Ref. [3] where additional scalar fields, others than the electroweak Higgs doublet, participate in mass generation.

${ }^{\dagger}$ This is to avoid problems with weak interactions universality. We suppose that the Standard Model gauge fields are localized inside the brane by means of a mechanism [9, 10] which preserves charge universality by itself, see discussion in Ref. 11]. 
below), leaving only $n-2$ degenerate massless $\nu_{L}$ states. Since one strictly massless neutrino is no problem (only differences in masses squared are tested this far), the neutrino degeneracy is thus solved for $n \leq 3$.

The scheme presented below uses one extra scalar field to match quantum numbers and generate the neutrino masses. It is of course also possible to induce mixing terms, but these are probably redundant, since off-diagonal terms are already allowed for in the charged leptons sector.

\section{Models with a single generation in extra dimen- sions}

If different fermionic modes have different wave function profiles in extra dimensions, then their overlaps with the Higgs wave function may produce hierarchical structure of masses and mixings [1]. In the class of models [2, 3], each multi-dimensional fermion develops three chiral zero modes localized on a four-dimensional brane due to topological properties of the brane background. The Index theorem guarantees that the three zero modes are linearly independent, and thus have different profiles in extra dimensions. Analysis of the equations for these zero modes demonstrates that a hierarchy in the mass matrix indeed appears due to overlaps of the wave functions. For the discussion of this mechanism and comparison with other approaches, see Ref. [2].

We use conventions of Ref. [2] throughout the paper. We will work in a specific realisation of this approach which was suggested in Ref. [3]. The model is formulated in six dimensions, and the brane is an Abelian vortex made of the gauge field of $U(1)_{g}$ gauge group and a scalar field $\Phi$. Field content of the model of Ref. [3] is given in Table 1 for easy reference (we concentrate here on the leptonic sector since we are interested in neutrino masses). Like other Standard Model fermions, leptons are zero modes of the six-dimensional Dirac spinors, namely, of the electroweak $S U(2)$ doublet $L$ and $S U(2)$ singlet $E$ which have axial charges $+3 / 2$ and $-3 / 2$, respectively, under $U(1)_{g}$. The interaction with vortex field,

$$
g_{l} \Phi^{3} \bar{L} \frac{1-\Gamma_{7}}{2} L+g_{e} \Phi^{* 3} \bar{E} \frac{1-\Gamma_{7}}{2} E+\text { h.c. }
$$




\begin{tabular}{|c|c|c|c|c|c|}
\hline \multirow[t]{2}{*}{ fields } & \multirow[t]{2}{*}{ profiles } & \multicolumn{2}{|c|}{ charges } & \multicolumn{2}{|c|}{ representations } \\
\hline & & $U(1)_{g}$ & $U(1)_{Y}$ & $S U(2)_{W}$ & $S U(3)_{C}$ \\
\hline scalar $\Phi$ & $\begin{array}{c}F(r) \mathrm{e}^{i \theta} \\
F(0)=0, F(\infty)=v_{\Phi}\end{array}$ & +1 & 0 & 1 & 1 \\
\hline scalar $X$ & $\begin{array}{c}X(r) \\
X(0)=v_{X}, X(\infty)=0\end{array}$ & +1 & 0 & 1 & 1 \\
\hline scalar $H$ & $\begin{array}{c}H(r) \\
H(0)=v_{H}, H(\infty)=0\end{array}$ & -1 & $+1 / 2$ & 2 & 1 \\
\hline fermion $\quad L$ & $3 \mathrm{~L}$ zero modes & axial $+3 / 2$ & $-1 / 2$ & 2 & 1 \\
\hline fermion $E$ & $3 \mathrm{R}$ zero modes & axial $-3 / 2$ & -1 & 1 & 1 \\
\hline
\end{tabular}

Table 1: Field content of the model of Ref. [3] (scalars and leptons only).

results in three localized left- (right-)handed in four-dimensional sence zero modes of $L(E)$ which describe three generations of usual leptons. The zero modes have the form

$$
L_{p} \sim\left(\begin{array}{c}
0 \\
\mathbf{l}_{p} l_{p}(r) \mathrm{e}^{i p \theta} \\
\mathbf{l}_{p} l_{2-p}(r) \mathrm{e}^{-i(2-p) \theta} \\
0
\end{array}\right), \quad E_{p} \sim\left(\begin{array}{c}
\mathbf{e}_{p} e_{2-p}(r) \mathrm{e}^{-i(2-p) \theta} \\
0 \\
0 \\
\mathbf{e}_{p} e_{p}(r) \mathrm{e}^{i p \theta}
\end{array}\right),
$$

where $r, \theta$ are polar coordinates in the bulk, $p=0,1,2$ enumerates three zero modes which in the effective four-dimensional Lagrangian are described by two-component Weyl spinors $\mathbf{l}_{p}, \mathbf{e}_{p}$, left- and right-handed, respectively. The radial functions $L_{p}(r)$, $e_{p}(r)$ have the following leading behaviour:

$$
l_{p}(r) \sim r^{p}, r \rightarrow 0 ; \quad l_{p}(r) \sim \mathrm{e}^{-g_{l} v r}, r \rightarrow \infty,
$$

and the similar for $e_{p}(r)$.

The scalar potential

$$
\frac{\lambda}{2}\left(|\Phi|^{2}-v^{2}\right)^{2}+\frac{\kappa}{2}\left(|H|^{2}-\mu^{2}\right)^{2}+h^{2}|H|^{2}|\Phi|^{2}+\frac{\rho}{2}\left(|X|^{2}-v_{1}^{2}\right)^{2}+\eta^{2}|X|^{2}|\Phi|^{2}
$$

results in localisation of $H$ and $X$ inside the vortex. Interaction with the Higgs field,

$$
Y H X \bar{L} \frac{1-\Gamma_{7}}{2} E+Y \epsilon H \Phi \bar{L} \frac{1-\Gamma_{7}}{2} E+\text { h.c., }
$$




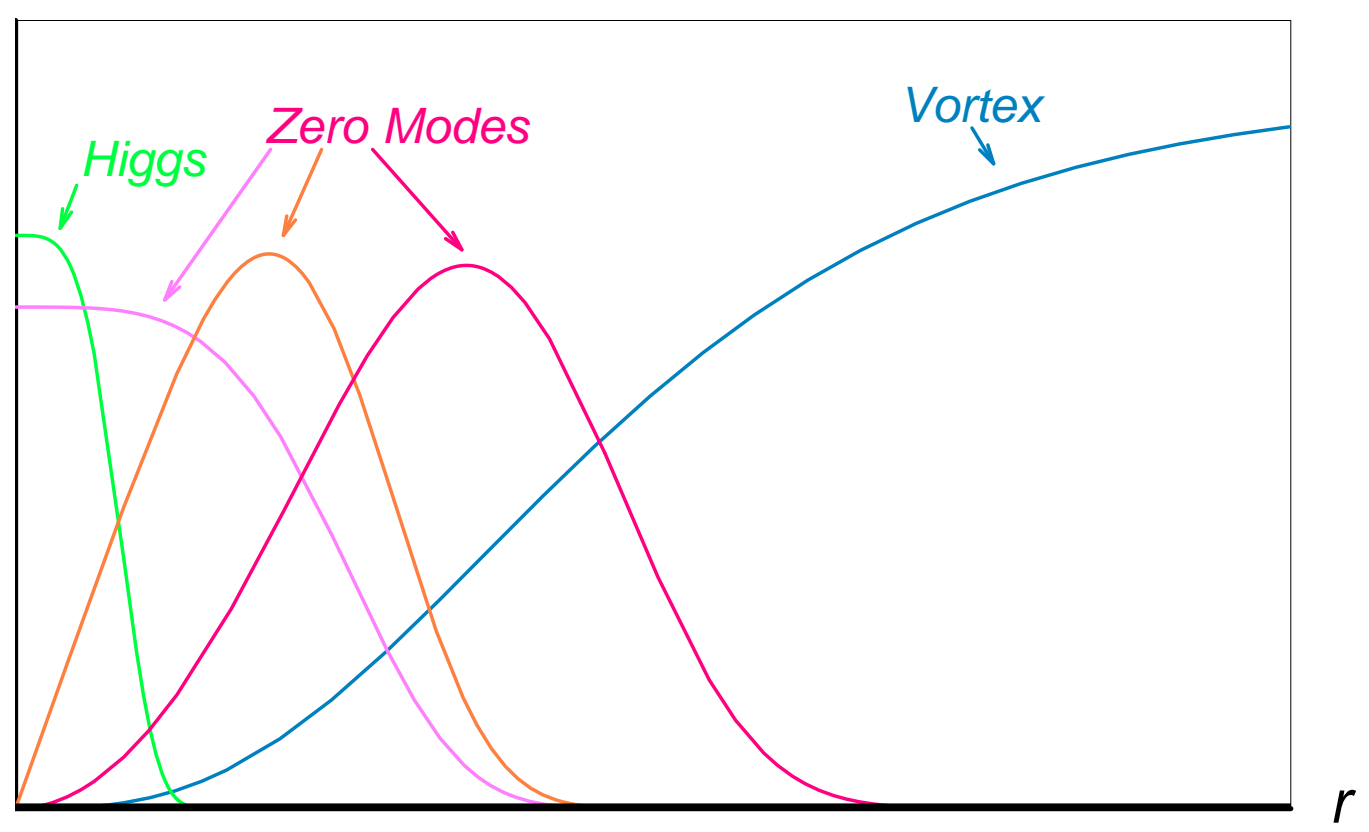

Figure 1: Sketch of the wave function profiles inside the brane.

where $Y$ and $Y \epsilon$ are two Yukawa couplings, provides a hierarchical structure of masses and mixings of charged leptons, as is discussed in Refs. [2, 3]. The reason for hierarchy is the different behaviour of three zero modes inside the brane which, after integrating out extra dimensions, results in the hierarchical structure of overlaps of wave functions for relatively narrow Higgs field (see the sketch at Fig. 1).

\section{$3 \quad$ Neutrino masses}

At the next step we follow the usual approach [5] to neutrino masses in models with extra dimensions, namely, we put a singlet fermion in the bulk. It plays the role of the usual right-handed neutrino of the see-saw models. The Dirac masses of the localized neutrinos are suppressed by the bulk size. The usual approach is to put three singlets in the bulk, otherwise only one linear combination of localized modes gets mass. This is in some contradiction with our intent to have a single generation in the higher dimensions. However, with more than one extra dimensions the bulk spinor has, in principle, enough components to give masses to more than one fourdimensional neutrino in the effective theory $[12$. Here, we exploit this fact in a slight 
modification of our model of Ref. [3].

Let us introduce the six-dimensional Dirac spinor $N$, which is a Standard Model singlet and has no half-integer axial charge under $U(1)_{g}$. In this case, no interaction term similar to Eq. (1), which could result in localisation of the zero modes of $N$, can be written. The free spinor $N$ satisfies the six-dimensional Dirac equation,

$$
i \Gamma_{A} \partial_{A} N=0
$$

It is convenient to perform the four-dimensional Fourier transform first,

$$
N=\int d^{4} k \mathrm{e}^{-i k^{\mu} x_{\mu}} \tilde{N}\left(K_{\mu}, r, \theta\right)
$$

substitute to the Dirac equation and multiply the latter by $\Gamma_{0}$,

$$
\left(k_{0}-k_{i} \Gamma_{0} \Gamma_{i}\right) \tilde{N}=D \tilde{N}
$$

where the operator $D=i \Gamma_{0} \Gamma_{\alpha} \partial_{\alpha}$ anticommutes with the operator in the left hand side. The latter fact allows us to look for a solution expanded in series of eigenvectors of $D$ with eigenvalues $\omega$,

$$
D \tilde{N}=\omega \tilde{N}
$$

The solution to this equation can be found in Appendix A. We note here that the zero mode $\omega=0$ which is regular both in the origin and at infinity consists of just four linearly independent constant two-component spinors,

$$
\tilde{N}=\left(\begin{array}{l}
\mathbf{n}_{1} \\
\mathbf{n}_{2} \\
\mathbf{n}_{3} \\
\mathbf{n}_{4}
\end{array}\right) .
$$

From the four-dimensional point of view, we have two left-handed Weyl spinors $\mathbf{n}_{2,3}$ and two right-handed Weyl spinors $\mathbf{n}_{1,4}$ (see discussion of chirality in six and four dimensions in ref. [2]). The latter two independent spinors, indeed, can be used to give masses to two of three localized neutrinos. This provides three $\Delta m^{2}$ which appear in the usual four-dimensional neutrino models.

The advantage of having an extra Weyl spinor, however, disappears if we use interactions with $H, X$, and $\Phi$, similar to Eq.(2). Indeed, $U(1)_{g}$ invariance requires 


\begin{tabular}{|c|c|c|c|c|c|}
\hline \multirow[t]{2}{*}{ fields } & \multirow[t]{2}{*}{ profiles } & \multicolumn{2}{|c|}{ charges } & \multicolumn{2}{|c|}{ representations } \\
\hline & & $\overline{U(1)_{g}}$ & $\overline{U(1)_{Y}}$ & $\overline{S U(2)_{W}}$ & $S U(3)_{C}$ \\
\hline fermion $N$ & bulk modes & +1 & 0 & 1 & 1 \\
\hline scalar $S$ & $\begin{array}{c}S(r) \\
S(0)=v_{S}, S(\infty)=0\end{array}$ & $+3 / 2$ & 0 & 1 & 1 \\
\hline
\end{tabular}

Table 2: Fields introduced to give masses to the neutrinos.

that $N$ has the charge $3 / 2$ under this group (half-integer axial charges are not allowed in order not to trap the $N$ particle on the brane) for the term like

$$
H X \bar{L} \frac{1-\Gamma_{7}}{2} N
$$

(or $-3 / 2$ if we use $\left(1+\Gamma_{7}\right) / 2$ ). Without the projector $\left(1 \pm \Gamma_{7}\right) / 2$, the term is not gauge invariant; on the other hand, this projector kills one of two spinors $\mathbf{n}_{1,4}$. To overcome this difficulty, we introduce a new scalar field $S$ which is analogous to $X$ but has a charge $1 / 2$ under $U(1)_{g}$ (the two fields, $N$ and $S$, which we introduce in this paper, are described in Table 2 . With these charge assignements, we can write down two interaction terms,

$$
y_{1} H S \bar{L} \frac{1-\Gamma_{7}}{2} N
$$

and

$$
y_{2} H S^{*} \bar{L} \frac{1+\Gamma_{7}}{2} N
$$

Substituting zero modes of $l$ and $N$, one gets the effective Lagrangian

$$
\int r d r d \theta\left(y_{1} H S(r) l_{2-p}(r) \mathrm{e}^{-i(2-p) \theta} \mathbf{l}_{p} \mathbf{n}_{1}+y_{2} H S(r) l_{p}(r) \mathrm{e}^{i p \theta} \mathbf{l}_{p} \mathbf{n}_{4}\right)
$$

Since $H$ and $S$ have no $\theta$ dependence in their classical profiles, the integral over $\theta$ is non-zero for $p=2$ (the first term) and $p=0$ (the second). Thus, the modes $\mathbf{l}_{0,2}$ and

${ }^{\ddagger}$ With these charge assignments, Dirac mass term for $N$ is not forbidden. The simplest way to forbid it is to assign a mixed, axial and vector, charge to $N$. The axial charge $q_{A}$ should not be half-integer, otherwise $N$ is localised on the brane. Together with the charge $q_{S}$ of $S$, they satisfy $q_{S}=3 / 2+q_{A}$, while the vector charge of $N, q_{V}=1$. For example, one of the solutions is $q_{A}=1 / 4$, $q_{V}=1, q_{S}=7 / 4$. 
$\mathbf{n}_{4,1}$ are paired in the mass matrix. The masses are given by integrals

$$
y_{1,2} 2 \pi \int r d r H S(r) l_{0}(r)\left|n_{4,1}\right|
$$

where the norm $\left|n_{i}\right| \sim 1 / R, R$ being the size of the extra dimensions. Masses of $\mathbf{l}_{0}$ and $\mathbf{l}_{2}$ differ only by Yukawa couplings $y_{1,2}$ in two terms.

Two notes are in order. First, would we add another scalar with the same quantum numbers as $S$ but with winding number 1, we would receive a more complicated mass matrix (mixing would appear in the neutrino sector as well as in the sector of charged leptons), but not the mass for the third neutrino - because we have only two righthanded Weyl spinors, $\mathbf{n}_{1}$ and $\mathbf{n}_{4}$, available. One linear combination of $\mathbf{l}_{p}$ would still be massless. Second, the Eqs. (田), (国) mix $\mathbf{l}_{p}$ with nonzero modes of $N$ as well. However, to satisfy simultaneously constraints from weak charge universality and from Supernova 1987a, these mixings should be negligible.

\section{Parameters and estimates}

In order to count parameters and to estimate the scales involved in the model, we make use of the "step Higgs" approximation (full numerical analysis will be presented elsewhere [13]). First of all, recall that in six dimensions, a scalar field has dimension $(\text { mass })^{2}$ and a fermion field has dimension $(\text { mass })^{5 / 2}$. This means that the transverse part of the fermion, described by $l_{p}(r)$, has dimension (mass) ${ }^{1}$ (four-dimensional spinors, denoted by letters in bold face, are of course $\left.(\text { mass })^{3 / 2}\right)$. Dimensions of cou-

pling constants are $[Y]=(\text { mass })^{-3},[g]=(\text { mass })^{-5},[h]=(\text { mass })^{-1},[\lambda]=(\text { mass })^{-2}$. The fundamental dimensionful parameters of the theory are the vortex scale $v$ (in theories where large extra dimensions are invoked to reformulate the gauge hierarchy problem, $v$ is of order multi-dimensional Planck mass), and the compactification scale $R$. It is convenient to rescale the parameters of the theory:

$$
\tilde{g}_{l, e}=g_{l, e} v^{5 / 2}, \tilde{v}=v^{1 / 2}, \tilde{\mu}=\mu v^{-1 / 2}, \quad \tilde{v}_{1}=v_{1} v^{-1 / 2}, \tilde{h}=h v^{1 / 2}, \tilde{Y}=Y v^{3 / 2}, \tilde{\lambda}=\lambda v
$$

Tilded coupling constants $\tilde{g}, \tilde{h}, \tilde{Y}, \tilde{\lambda}$ are dimensionless, while $\tilde{v}, \tilde{v}_{1}, \tilde{\mu}$ have dimension (mass $)^{1}$. 
In the narrow Higgs approximation, $H(r)$ can be substituted by

$$
H(r) \simeq\left\{\begin{array}{cc}
H(0), & r \leq r_{H} \\
0, & r>r_{H}
\end{array}\right.
$$

where $r_{H} \sim(\tilde{h} \tilde{v})^{-1}$ is the width of $H(r)$ which is supposed to be smaller than widths of other profiles. Then, we can use the following approximations for $r<r_{H}$ :

$$
\begin{gathered}
X(r) \approx X(0), \quad S(r) \approx S(0), \\
l_{p}(r) \approx \sqrt{\tilde{g}_{l}} \tilde{v}\left(\sqrt{\tilde{g}_{l}} \tilde{v} r\right)^{p}, \\
e_{p}(r) \approx \sqrt{\tilde{g}_{e}} \tilde{v}\left(\sqrt{\tilde{g}_{e}} \tilde{v} r\right)^{p},
\end{gathered}
$$

The coefficients at $r^{p}$ in fermionic radial functions are determined, approximately, in Ref. [13]. For the scalar fields $H, X$, and $S$, the boundary conditions at $r=0$ are $\partial H(r) / \partial r=0$, etc.; so that $H(0), X(0), S(0)$ are to be determined by solving the full nonlinear system [13]. We denote $H(0)=v_{H}, X(0)=x v_{H}, S(0)=s v_{H}$, where $x$ and $s$ are coefficients of order one, $v_{H}$ depends non-trivially on $\mu$ and $v$. Since $\mu$ and $v$ are independent parameters of the lagrangian, $v_{H}$ does not need to be very close to $v$, though we do not wish them to differ too much in order to avoid fine tuning of the parameters.

Diagonal elements of the mass matrix of charged leptons are, to this approximation,

$$
m_{p p} \approx 2 \pi Y H(0) X(0) \int_{0}^{r_{H}} r d r l_{p}(r) e_{p}(r) \approx \pi \tilde{Y} x \frac{\tilde{v}_{H}^{2}}{\tilde{v}} \frac{\delta^{p+1}}{p+1},
$$

where $\delta=\sqrt{\tilde{g}_{l} \tilde{g}_{e}} / \tilde{h}^{2}$ should be sufficiently small to provide the mass hierarchy between three generations. Smallness of $\delta$ exactly corresponds to the fact that $H(r)$ is more narrow than $l(r), e(r)$.

Non-diagonal mass matrix elements are estimated in a similar way, taking into account the behaviour of $F(r)$ at small $r$ :

$$
F(r) \sim v(\sqrt{\tilde{\lambda}} v r)
$$

The result is that

$$
m_{p, p-1} \approx m_{p p} \tilde{\epsilon}
$$


where generation-independent constant

$$
\tilde{\epsilon}=\frac{\epsilon}{x} \frac{\tilde{v}}{\tilde{v}_{H}} \sqrt{\frac{\tilde{\lambda}}{\tilde{g}_{e}}} .
$$

This corresponds to the mass matrix of charged leptons

$$
M_{e} \approx M_{0 e}\left(\begin{array}{ccc}
1 & 0 & 0 \\
\tilde{\epsilon} & \delta / 2 & 0 \\
0 & \tilde{\epsilon} \delta / 2 & \delta^{2} / 3
\end{array}\right)
$$

where the overall constant $M_{0 e}=\pi x \tilde{Y} \tilde{v}_{H}^{2} / \tilde{v}$.

It is straightforward to obtain, to the same approximation, the neutrino mass matrix

$$
M_{\nu}=M_{0 \nu}\left(\begin{array}{cc}
1 & 0 \\
0 & 0 \\
0 & \tilde{y}_{2} / \tilde{y}_{1}
\end{array}\right)
$$

where

$$
M_{0 \nu}=\sqrt{\pi} \tilde{y}_{1} \frac{\tilde{g}_{l}}{\tilde{h}^{2}} \frac{\tilde{v}_{H}^{2}}{\tilde{v}} \frac{1}{\tilde{v} R}
$$

and we rescaled, as before, $\tilde{y}_{1,2}=y_{1,2} v^{3 / 2}$. The matrix is $3 \times 2$ because we have only two light right-handed modes, $\mathbf{n}_{1}$ and $\mathbf{n}_{4}$.

Thus, up to dimensionless constants of order one, the neutrino mass scale is suppressed by a factor of $\frac{1}{\tilde{v} R}$ with respect to the mass scale of charged fermions, $\tilde{v}_{H}^{2} / \tilde{v}$. One can have simultaneously $\tilde{v}_{H}^{2} / \tilde{v} \sim 100 \mathrm{GeV}, m_{\nu} \sim 0.1 \mathrm{eV}$, and Planck mass relation $\tilde{v}^{2} R \sim 10^{19} \mathrm{GeV}$ satisfied for $\tilde{v} \sim 1000 \mathrm{TeV}, \tilde{v}_{H} \sim 10 \mathrm{TeV}, R \sim 10^{-6} \mathrm{~mm}$.

In our model, neutrino mass matrix does not contain mixings. In the sector of charged leptons, however, mixing appears, see Eq. (6). Three parameters $M_{0 e}, \delta$, and $\tilde{\epsilon}$, determine the masses of three charged leptons; one can fit known values of $M_{e, \mu, \tau}$ with $M_{0 e} \approx 650 \mathrm{MeV}, \delta \approx 0.13, \tilde{\epsilon} \approx 2.5$. The latter value means that mixing is not negligible in the leptonic sector. Since all leptonic mixings appear in Eq. (6), our model predicts values of the neutrino mixing angles which are implicitly determined by masses of charged leptons. In usual notations, they correspond to $\sin \theta_{12} \sim 0.14$, $\sin \theta_{13} \sim 0.37, \sin \theta_{23} \sim 0.99$. On the other hand, values of two $\Delta m^{2}$ are predicted only by the order of magnitude since they depend on unknown constants $\tilde{y}_{1,2}$. 


\section{Conclusions}

In a class of multidimensional models with one vector-like fermionic family, the lowenergy effective theory describes three chiral families in four dimensions. Hierarchy of fermionic masses appears without fine tuning of parameters. In this paper, we have shown that nondegenerate neutrino masses can be incorporated in a model of this class with the help of only one multidimensional Dirac fermion. One of three neutrinos is exactly massless 3 while masses of two others are parametrically close to each other. Neutrino mass matrix is diagonal, but mixings in the leptonic sector are present in the mass matrix of charged leptons. Once masses of $e, \mu, \tau$ are fixed to their experimental values, the model predicts neutrino mixing angles, $\sin \theta_{12} \sim 0.54$, $\sin \theta_{13} \sim 0.31, \sin \theta_{23} \sim 0.99$. Of course, these values were obtained with very rough approximations, and should not be taken too seriously. Neutrino mass differences are predicted by order of magnitude only, $\Delta m^{2} \sim(0.1 \mathrm{eV})^{2}$ for natural values of parameters.

The authors are indebted to K.Agashe, E.Akhmedov, T.Gherghetta, M.Giovannini, I.Gogoladze, A.Neronov, E.Nugaev, V.Rubakov, G.Senjanovic, M.Shaposhnikov, S.Sibiryakov, A.Smirnov for helpful discussions on different subjects related to this work. The work of M.L. and S.T. is supported in part by RFFI grant 99-02-18410a, by the Council of Presidential Grants and State Support of Leading Scientific Schools, grant 00-15-96626, by CRDF award RP1-2103, and by the programme SCOPES of the Swiss National Science Foundation, project No. 7SUPJ062239, financed by Federal Department of Foreign affairs. The work of S.T. is supported in part by Swiss Science Foundation, grant 21-58947.99. This work was initiated during the visits of M.L. and S.T. to ULB, which we thank for kind hospitality and partial support by the "Actions de Recherche Concretées" of "Communauté Française de Belgique" and IISN-Belgium. S.T. thanks High Energy Group of ICTP (Trieste) for hospitality during his visit, when part of this work was done and numerous discussions were carried out.

\footnotetext{
${ }^{\S}$ See, however, Appendix B
} 


\section{A Solution to the Dirac equation for $N$}

In this Appendix, we present the solution to the transverse part of Dirac equation,

$$
D \tilde{N}=\omega \tilde{N}
$$

where $D=i \Gamma_{0} \Gamma_{\alpha} \partial_{\alpha}$. The general solution for $\omega=0$ is

$$
\tilde{N}=\left(\begin{array}{c}
\frac{1}{\mathcal{N}_{1}} \mathbf{n}_{1} r^{-p_{1}} \mathrm{e}^{i p_{1} \theta} \\
\frac{1}{\mathcal{N}_{2}} \mathbf{n}_{2} r^{p_{2}} \mathrm{e}^{i p_{2} \theta} \\
\frac{1}{\mathcal{N}_{3}} \mathbf{n}_{3} r^{-p_{3}} \mathrm{e}^{i p_{3} \theta} \\
\frac{1}{\mathcal{N}_{4}} \mathbf{n}_{4} r^{p_{4}} \mathrm{e}^{i p_{4} \theta}
\end{array}\right) .
$$

Here, $\mathbf{n}_{a}$ are independent two-component spinors. The solutions regular both at small and large $r$ correspond to $p_{a}=0$, Eq. (3). For them, the normalization factors $\mathcal{N}_{a}=\sqrt{\pi} R$, where $R$ is the radius of the bulk.

For $\omega \neq 0$, the regular at $r=0$ solution depends on two two-component spinors $\mathbf{n}, \mathbf{m}$ for each $\omega$ :

$$
\tilde{N}=\left(\begin{array}{c}
\mathbf{n}_{\omega} J_{p}(\omega r) \mathrm{e}^{i p \theta} \\
-\mathbf{n}_{\omega} J_{p-1}(\omega r) \mathrm{e}^{i(p-1) \theta} \\
\mathbf{m}_{\omega} J_{q}(\omega r) \mathrm{e}^{i q \theta} \\
\mathbf{m}_{\omega} J_{q-1}(\omega r) \mathrm{e}^{i(q-1) \theta}
\end{array}\right)
$$

A series of eigenvalues $\omega_{n}^{(p)}$ are determined by the boundary conditions at large $r$. The exact eigenvalues depend on the compactification scheme chosen (see discussion in Appendix B), but in general, they are of order

$$
\omega_{n}^{(p)} \sim \pi n / R
$$

\section{B Compactification}

The compactification scheme defines boundary conditions at large $r$. It should satisfy the following criteria:

(i) it should preserve three zero modes with different winding numbers of the fields localized on the vortex;

(ii) it should allow for at least one (Dirac) zero mode of the bulk singlet. 
The second requirement is essentially equivalent to compactification on a Ricci-flat manifold (that is, a manifold with zero scalar curvature $\mathcal{R}$ ) [14. Toroidal compactifications satisfy this requirement since the torus have $\mathcal{R}=0$. However, the corresponding boundary conditions usually kill one or two of three zero modes because they allow either even or odd winding numbers for a given field. Compactification of a vortex on a sphere preserves three zero modes [15] but does not satisfy the second requirement: for a sphere, $\mathcal{R} \neq 0$. This would open an interesting possibility of having three massless neutrinos and explaining observed neutrino anomalies by mixing with Kaluza-Klein modes only (this would lead to a nonconventional pattern of oscillations, as was discussed in Ref. [16] in the frameworks of a toy model). However, this possibility is in contradiction with bounds on mixing with Kaluza-Klein states from supernova evolution.

One can also keep the compactification mechanism unspecified, and consider flat space of finite volume. This means that the fields will live inside a cilinder of large radius $R$ in six dimensions. The corresponding boundary condition, that is, absence of fermionic current through the boundary, can be reformulated [17] as

$$
i \Gamma_{A} \partial_{A} \psi=\psi
$$

for a Dirac spinor $\psi$. Applied to $N$, Eq. (7), this boundary condition selects not only constant, but also rather peculiar zero modes which grow up at large $r$. In this case, it is not necessary to introduce the additional field $S$ to obtain a second neutrino mass, and it suffices to use the additional mode, alternatively, with the field $S$ included, 3 (even 4 if the model is extended) neutrino masses can be generated, although the last 2 are then hierarchically smaller than the ones previously defined. At this level however, such modes appear more as a quirk of a particular way to implement the finite volume.

It seems the most promising to have a finite volume in the case of warped transverse dimensions. Particularly interesting is the fact that there exist six-dimensional solutions with gravity localized on an Abelian vortex, Ref. [18. These six-dimensional solutions provide also a natural framework for the localization of gauge fields at weak coupling, thus opening the way to fully realistic and calculable models. 


\section{References}

[1] N. Arkani-Hamed and M. Schmaltz, Phys. Rev. D61 (2000) 033005 hepph/9903417;

G. Dvali and M. Shifman, Phys. Lett. B475 (2000) 295 hep-ph/0001072;

T. Gherghetta and A. Pomarol, hep-ph/0003129;

D. E. Kaplan and T. M. Tait, JHEP 0006 (2000) 020 hep-ph/0004200;

S. J. Huber and Q. Shafi, hep-ph/0010195.

[2] M. V. Libanov and S. V. Troitsky, Nucl. Phys. B 599, 319 (2001) hep$\mathrm{ph} / 0011095$.

[3] J. M. Frere, M. V. Libanov and S. V. Troitsky, Phys. Lett. B 512, 169 (2001) hep-ph/0012306.

[4] E. J. Weinberg, Phys. Rev. D24 (1981) 2669;

[5] N. Arkani-Hamed, S. Dimopoulos, G. Dvali and J. March-Russell, hepph/9811448;

K. R. Dienes, E. Dudas and T. Gherghetta, Nucl. Phys. B 557 (1999) 25 hep$\mathrm{ph} / 9811428$.

[6] G. Barenboim, G. C. Branco, A. de Gouvea and M. N. Rebelo, hep-ph/0104312.

[7] A. De Gouvea, G. F. Giudice, A. Strumia and K. Tobe, hep-ph/0107156.

[8] R. Barbieri, P. Creminelli and A. Strumia, Nucl. Phys. B 585 (2000) 28 hepph/0002199;

A. Lukas, P. Ramond, A. Romanino and G. G. Ross, JHEP 0104 (2001) 010 hep-ph/0011295.

[9] G. Dvali and M. Shifman, Phys. Lett. B 396 (1997) 64 [Erratum-ibid. B 407 (1997) 452] hep-th/9612128.

[10] E. K. Akhmedov, hep-th/0107223.

[11] S. L. Dubovsky and V. A. Rubakov, hep-th/0105243. 
[12] K. Agashe and G. Wu, Phys. Lett. B 498 (2001) 230 [hep-ph/0010117.

[13] E.Ya.Nugaev, Master Thesis from the Moscow Institute of Physics and Technology, 2001; M.V.Libanov and E.Ya.Nugaev, to appear.

[14] E. Witten, "Fermion Quantum Numbers In Kaluza-Klein Theory," PRINT-831056 (Princeton). In: Appelquist, T. (Ed.) et al.: Modern Kaluza-Klein Theories, 438-511.

[15] A. Nakamura, S. Hirenzaki and K. Shiraishi, Nucl. Phys. B 339 (1990) 533;

A. Nakamura and K. Shiraishi, Mod. Phys. Lett. A 5 (1990) 1109.

[16] N. Cosme, J. M. Frere, Y. Gouverneur, F. S. Ling, D. Monderen and V. Van Elewyck, Phys. Rev. D 63 (2001) 113018 [hep-ph/0010192].

[17] K. Johnson, Acta Phys. Polon. B 6 (1975) 865.

[18] T. Gherghetta and M. Shaposhnikov, Phys. Rev. Lett. 85 (2000) 240 hepth/0004014;

M. Giovannini, H. Meyer and M. Shaposhnikov, hep-th/0104118. 